\title{
Predição do tempo de vida de baterias utilizadas em dispositivos móveis: modelagem matemática e validação de modelos baseados em cinética e difusão unidimensional
}

\author{
Douglas Joziel Bitencourt Freitas 1 \\ Paulo Sérgio Sausen ${ }^{1}$ \\ Airam Teresa Zago Romcy Sausen ${ }^{1}$
}

\begin{abstract}
Resumo: Neste artigo são apresentadas a modelagem matemática e a validação de dois modelos analíticos: Modelo Cinético de Bateria e Modelo de Difusão de Rakhmatov e Vrudhula, ambos empregados para predição do tempo de vida de baterias de Li-Po, utilizadas em dispositivos móveis, uma vez que esses modelos não foram desenvolvidos com foco em baterias de pequena capacidade. O interesse pela aplicação dos modelos analíticos ocorre devido a sua simplicidade matemática, e por capturarem dois efeitos não lineares importantes, a taxa de capacidade e o efeito de recuperação, a partir de equações diferenciais solúveis por métodos analíticos tradicionais. Destaca-se que esse trabalho objetiva fornecer aos projetistas de smartphones modelos matemáticos acurados, que podem ser utilizados para aperfeiçoar os Sistemas de Gerenciamento de Energia desses aparelhos. Além disso, diferentemente de outros trabalhos da literatura que empregam perfis de correntes de descarga constantes ao longo do tempo, esta pesquisa visa representar um cenário realístico, a partir de perfis de correntes de descarga variáveis. Os modelos foram simulados e validados por comparação entre os resultados das simulações computacionais e os dados experimentais de descargas de baterias, obtidos a partir de uma plataforma de testes especialmente construída para a coleta de dados. Os resultados das simulações comprovam que o Modelo de Difusão obteve o menor erro médio de $1,55 \%$, quando comparado aos demais modelos analisados na predição do tempo de vida de baterias do tipo Li-Po.
\end{abstract}

Palavras-chave: Modelagem matemática. Modelos analíticos de baterias. Tempo de vida de baterias.

\begin{abstract}
This paper presents the mathematical modeling and the validation of two analytical models : Kinetic Battery Model and Diffusion Model of Rakhmatov and Vrudhula, both employed to predict the batteries lifetime of Li-Po used in mobile devices, since these models were not developed with focus on small capacity batteries. The interest in the application of analytical models occurs due to its mathematical simplicity, and by capturing two important nonlinear effects, the capacity rate and the recovery effect, from differential equations soluble by traditional analytical methods. It stands out that this paper aims to provide the designers of smartphones accurate mathematical models that can be used to improve the Energy Management Systems of these devices. Moreover, unlike other works of the literature which employ profiles of constant discharge currents over time, this research aims to represent a realistic scenario, from profiles of variable discharge currents. The models were simulated and validated by a comparison between the computational simulations results and the experimental data of battery discharges obtained from a test platform specially constructed for data collection. The results of the simulations show that the diffusion model obtained the lowest average error of $1.55 \%$, when compared to the other models analyzed in the prediction of the batteries lifetime of Li-Po.
\end{abstract}

Keywords: Mathematical modeling. Analytical battery models. Battery lifetime.

\footnotetext{
${ }^{1}$ Programa de Pós-Graduação em Modelagem Matemática (PPGMM), Unijuí, R. Lulu Ingelfritz, 480 - Ijuí (RS) - Brasil \{douglasjozielemx2.unisc.br, sausen@unijui.edu.br, airam@unijui.edu.br\}
}

http://dx.doi.org/10.5335/rbca.v9i4.7136

Revista Brasileira de Computação Aplicada (ISSN 2176-6649), Passo Fundo, v. 9, n. 4, p. 76-89, dez. 2017 


\section{Introdução}

Da primitiva pilha voltaica às modernas baterias de Lítio Íon (Li-Íon), as baterias eletroquímicas ainda são um paradigma tecnológico para o armazenamento portátil de energia elétrica. Disponíveis sob diversas configurações, atendendo demandas específicas de energia, as baterias são elementos fundamentais à industria na produção, em larga escala, de dispositivos móveis cada vez mais compactos. Dentre as aplicações em dispositivos móveis, destacam-se as baterias recarregáveis empregadas na alimentação de smartphones, as quais são o objeto de estudo deste artigo.

Por definição, uma bateria é um dispositivo formado por células eletroquímicas de elevado eletropotencial, que transforma a energia química, acumulada em seus materiais constituintes, em energia elétrica, por oxirredução [1]. As baterias possuem capacidade finita para o armazenamento de energia, necessitando de uma recarga após cada período de descarga. Assim, pode-se afirmar que a mobilidade operacional de um dispositivo móvel está condicionada, proporcionalmente, ao tempo de $\operatorname{vida}^{2}$ da bateria.

Em um smartphone o gerenciamento estratégico da energia disponível na bateria tem significativa relevância, sobretudo, por informar ao usuário o tempo que o dispositivo ainda se manterá operacional, até que seja necessária uma nova recarga. Nesse sentido, os dispositivos possuem por default um Sistema de Gerenciamento de Energia (SGE) que realiza, basicamente, o monitoramento do estado de carga (SOC) da bateria, predizendo o seu tempo de vida por meio de um modelo matemático. A exemplo do Android, que é o sistema operacional amplamente utilizado em smartphones [2], o modelo embarcado no SGE para a predição do tempo de vida é o Modelo Linear [3], o qual não leva em conta importantes efeitos não lineares, presentes no processo de descarga, que influenciam no tempo de vida da bateria.

O processo de descarga é resultante de complexas interações entre os processos físicos, químicos e efeitos não lineares que ocorrem no interior da bateria, o que torna a modelagem acurada dessa dinâmica um desafio. Durante a descarga, o desempenho da bateria em relação ao perfil de consumo depende, essencialmente, da corrente elétrica exigida pelo sistema combinada com dois importantes efeitos não lineares que são: a taxa de capacidade e o efeito de recuperação. O primeiro relaciona a corrente de descarga e a capacidade de absorção de íons pelo cátodo [1], o segundo descreve a reorganização do gradiente de concentração de íons no interior da bateria, sendo que ambos ocorrem de modo alternado [4].

Ao longo das últimas décadas, através do emprego da modelagem matemática, diversos estudos vêm propondo e aprimorando modelos, buscando descrever com acurácia a dinâmica do processo de descarga de baterias. Em linhas gerais, os modelos matemáticos, relatados frequentemente na literatura, estão fundamentados nos processos fenomenológicas tradicionais, centrados ora nas características físicas da estrutura da bateria [5], ora nas reações eletroquímicas intrínsecas ao processo de descarga [6]. Nesse cenário, evidenciam-se as classes dos modelos analíticos [7], autorregressivos [8], elétricos [5], eletroquímicos [6], estocásticos [9] e híbridos [10].

Além da diversidade de classes de modelos associados ao significativo número destes, nem todos são aplicáveis aos SGEs embarcados em smartphones, principalmente, pela complexidade das suas estruturas matemáticas e, por consequência, pela dificuldade em estimar seus parâmetros empíricos. Adiciona-se a esse fato, o emprego histórico do Modelo Linear de bateria por uma vasta gama de SGEs, inclusive, presentes em diversos modelos de dispositivos mais modernos [11]. É neste contexto, que o presente trabalho passa a ter uma significativa relevância e constitui-se em um terreno fértil à investigação da dinâmica do processo de descarga, a partir da modelagem matemática e da validação de modelos utéis aos SGEs, para realizar a predição acurada do tempo de vida de baterias de Li-Po.

Dentre as classes de modelos matemáticos de baterias, os analíticos destacam-se por descreverem a relação entre a capacidade da bateria e o tempo de vida, baseados em fenômenos empíricos ou em leis físicas análogas [7]. Desenvolvidos para baterias de alta capacidade, os modelos analíticos têm boa eficiência, com reduzido número de parâmetros, e são flexíveis à implementação computacional, requerendo a avaliação de expressões matemáticas analíticas, que podem ser reconfiguradas para diferentes tipos de baterias. Na literatura técnica, os principais modelos analíticos enfatizados são o Modelo Linear, a Lei de Peukert, o Modelo Cinético de Bateria e o Modelo de Difusão de Rakhmatov e Vrudhula [4].

\footnotetext{
${ }^{2}$ Neste artigo, define-se por tempo de vida a duração completa de um único ciclo de descarga total da bateria.
} 
Nesse panorama, o principal objetivo deste trabalho é apresentar a modelagem matemática e a validação de dois modelos analíticos - Modelo Cinético e Modelo de Difusão, para predição do tempo de vida de baterias de Lítio Polímero (Li-Po), utilizadas em smartphones. Diferentemente de outros trabalhos que empregam descargas hipotéticas, com perfis de corrente constantes ao longo do tempo, neste estudo busca-se representar um cenário realístico, utilizando perfis de correntes de descargas variáveis baseadas nas funcionalidades de um telefone celular do tipo smartphones. A escolha por estes modelos analíticos justifica-se, sobretudo, pela simplicidade matemática dos mesmos, e por capturarem os efeitos não lineares de taxa de capacidade e recuperação, a partir de equações diferenciais solúveis por métodos analíticos tradicionais.

A realização do trabalho se dá por meio de simulações computacionais dos modelos a partir da utilização de um extenso conjunto de ensaios, realizados em uma plataforma de testes desenvolvida especialmente para aquisição de dados empíricos do processo de descarga de baterias. Os resultados alcançados demonstram que os dois modelos analíticos avaliados e validados neste trabalho possuem melhor acurácia que o tradicional Modelo Linear. Sendo assim, este estudo contribui para o desenvolvimento estratégico de SGEs, visando o correto gerenciamento da energia disponível ao sistema operacional embarcado, estimando com precisão o tempo de vida da bateria e a operacionalidade do dispositivo.

O artigo está organizado da seguinte forma: Na Seção 2 são apresentadas generalidades sobre as baterias, os modelos matemáticos a serem empregados no trabalho e o desdobramento algébrico, em linhas gerais, das resoluções matemáticas de suas respectivas equações; na Seção 3 são esboçados os procedimentos metodológicos adotados para aquisição dos dados experimentais e para as simulações computacionais dos modelos; na Seção 4 os resultados são apresentados, seguidos de uma discussão e análise comparativa entre os modelos; e, por fim, na Seção 5 são traçadas as considerações finais e as perspectivas futuras da pesquisa.

\section{Modelagem matemática}

Nesta seção, inicialmente, são introduzidos alguns conceitos importantes acerca das baterias eletroquímicas, suas principais características e os efeitos não lineares recorrentes durante o processo de descarga. Em seguida, são descritos os modelos matemáticos a serem utilizados, incluindo, de modo sucinto, as equações que os compõem e os respectivos desdobramentos algébricos das soluções analíticas.

\subsection{Baterias}

As baterias são dispositivos que convertem energia química, acumulada em seus materiais ativos, em energia elétrica. Em geral, uma bateria é constituída por unidades básicas denominadas de células eletroquímicas, conforme Figura 1, conectadas em série e/ou paralelo. Durante o processo de descarga, a energia química de cada célula é transformada em energia elétrica por oxirredução - reversível por meio de recarga via fonte externa de energia elétrica [12].

A necessidade constante de melhorias no desempenho das baterias é, ainda hoje, um desafio de renovado interesse tecnológico [13]. Historicamente, a otimização das combinações entre os elementos químicos - chumbo, cádmio, níquel, lítio etc. - para o ânodo e o cátodo concorreu para o desenvolvimento das modernas baterias com estruturas compactas e capacidades ampliadas para o armazenamento de energia elétrica [12]. Embora distintos entre si, os tipos de baterias compartilham características comuns, as quais podem ser classificadas entre estáticas e dinâmicas [1], conforme a Tabela 1.

Por um lado, as características estáticas são definidas pela natureza eletroquímica e propriedades físicas da bateria; por outro, as características dinâmicas são aquelas que afetam diretamente nas condições de operacionalidade e no tempo de vida da bateria. Durante o processo de descarga são as características dinâmicas que sofrem a interferência dos efeitos não lineares, sendo essencial levar isso em consideração ao realizar a modelagem matemática dessa dinâmica, posto que esses efeitos influenciam - ou ampliando, ou reduzindo - o tempo de vida da bateria [1]. Na literatura, dois efeitos não lineares são recorrentes e possuem relevante impacto no processo de descarga: a taxa de capacidade e o efeito de recuperação [4].

Quando a corrente de descarga é reduzida (ou até mesmo nula), os íons se organizam uniformemente junto ao eletrodo. Porém, a partir do momento que uma corrente é extraída da bateria, os íons mais próximos do eletrodo

Revista Brasileira de Computação Aplicada (ISSN 2176-6649), Passo Fundo, v. 9, n. 4, p. 76-89, dez. 2017 
Figura 1: Esquema básico de uma célula de Li-Íon [13]

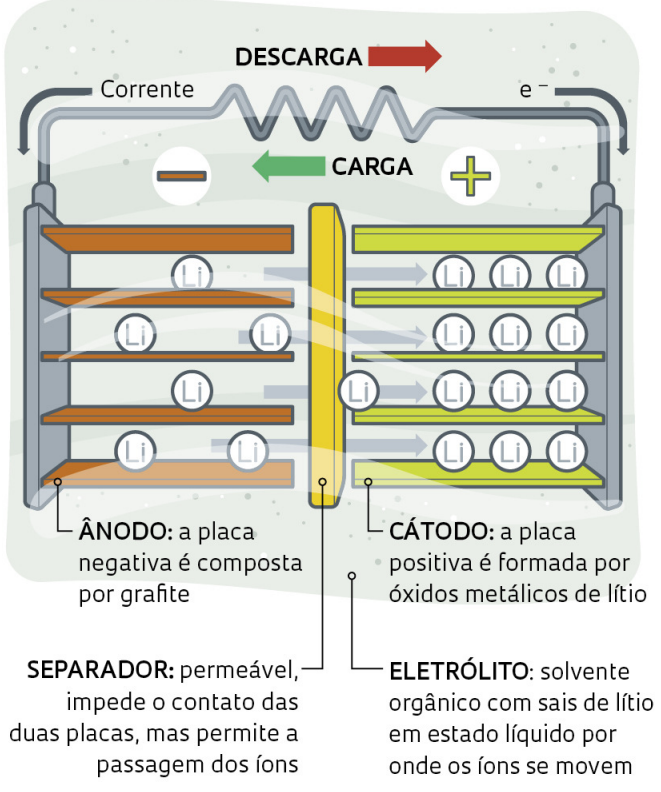

Tabela 1: Principais características das baterias

\begin{tabular}{cc}
\hline Estáticas & Dinâmicas \\
\hline Tensão nominal & Estado de carga \\
Nível de cut-off & Tensão de saída \\
Densidade de energia & Tensão de circuito aberto \\
Capacidade nominal & Temperatura Interna \\
Corrente máxima & \\
Taxa de autodescarga & \\
Ciclo de vida & \\
\hline
\end{tabular}

são utilizados, ocasionando um gradiente de íons ao longo do eletrólito. Como consequência, quanto maior for a corrente de descarga, maior será a capacidade indisponível da bateria, fenômeno conhecido como efeito da taxa de capacidade $[1,12]$.

No entanto, ao ocorrer uma redução significativa da corrente de descarga, os íons se reorganizam no interior da bateria. Essa reorganização, denominada efeito de recuperação, proporciona um sensível incremento na capacidade atual da bateria, refletindo inclusive na tensão de saída, visto que após a recuperação os íons encontram-se outra vez uniformemente distribuídos e disponíveis para a carga [1]. Até que a bateria atinja o nível de cut-off ${ }^{3}$ e seja considerada descarregada, os efeitos não lineares podem ocorrer repetidas vezes [4].

\subsection{Modelo Linear}

O Modelo Linear (ou Modelo Ideal) é considerado pela literatura técnica o mais simples dos modelos analíticos [14]. Analogamente, esse modelo representa um capacitor ideal, não levando em consideração os efeitos não lineares que ocorrem durante o processo de descarga, visto que a bateria é tratada como um recipiente linear de corrente. A capacidade restante $(C)$ de uma bateria, é dada pela equação

$$
C=C_{i}-I \cdot t_{d}
$$

${ }^{3} \mathrm{O}$ nível de cut-off é definido como a tensão mínima em que a bateria ainda pode fornecer energia ao sistema, após esse limite a bateria é considerada descarregada.

Revista Brasileira de Computação Aplicada (ISSN 2176-6649), Passo Fundo, v. 9, n. 4, p. 76-89, dez. 2017 
sendo que $C_{i}$ é a capacidade inicial da bateria (único parâmetro a determinar), $I$ é a corrente constante de descarga e $t_{d}$ é o tempo de duração da corrente aplicada [14].

Na equação (1), fazendo $C=0$, obtém-se o tempo de vida da bateria. Desta forma o tempo de duração da corrente de descarga equivale ao tempo de vida da bateria, ou seja, $t_{d}=L$. Logo, o tempo de vida $(L)$ de uma bateria para descargas constantes por meio do Modelo Linear é obtido a partir da equação

$$
L=\frac{C_{i}}{I} .
$$

Entretanto, em cenários reais a corrente nem sempre é plenamente constante, mas varia ao longo do tempo, conforme as operações do dispositivo. Nesse caso, a corrente de descarga $i(t)$ pode ser aproximada por um somatório de correntes constantes $I_{k}$, conforme apresentado na Figura 2. Considerando a função degrau $U(t)=1$, se $t \geq 0$, e $U(t)=0$, se $t<0$, a corrente variável $i(t)$ pode ser aproximada pela soma de $n$ degraus, dada pela expressão

$$
i(t) \approx \sum_{k=1}^{n} I_{k-1}\left[U\left(t-t_{k-1}\right)-U\left(t-t_{k}\right)\right]
$$

sendo que $I_{k}$ é a corrente constante de cada degrau, $I_{k-1}$ é a corrente de descarga durante o período $k-1$ e $U(t)$ é a função degrau definida como

$$
U(t)=\left\{\begin{array}{l}
1 \text { se } t \geq 0 \\
0 \text { se } t<0
\end{array}\right.
$$

Figura 2: Aproximação em degraus para corrente variável [14]

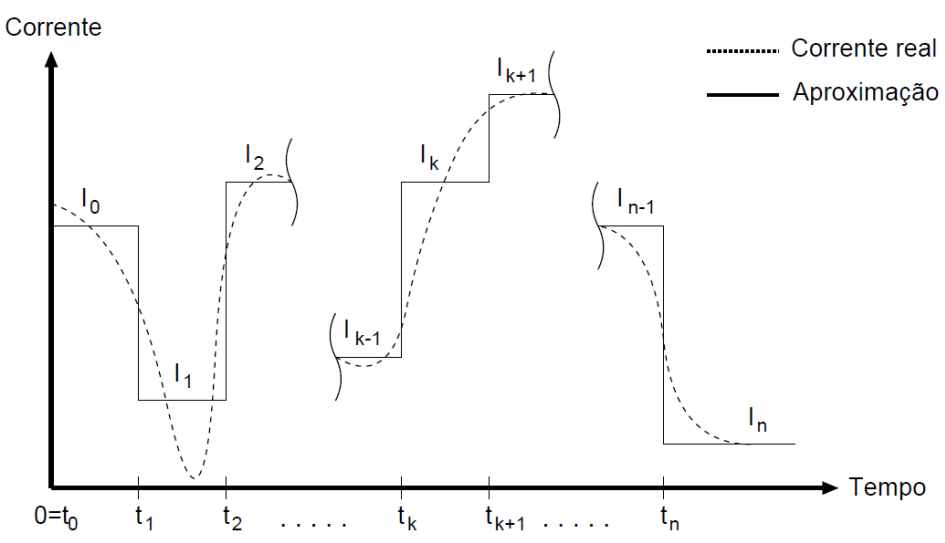

Assim, o tempo de vida de uma bateria, a partir do Modelo Linear, para perfis variáveis, é dado pela equação

$$
L=\frac{C_{i}}{\left[\frac{\sum_{k=1}^{n} I_{k-1}\left(t_{k}-t_{k-1}\right)}{L}\right]}
$$

sendo que $I_{k}$ é a corrente de descarga, $t_{k}$ é o tempo inicial da corrente de descarga, $t_{k-1}$ é o tempo final da corrente utilizada, e $C_{i}$ é o mesmo parâmetro estimado na equação (2) que está relacionado a capacidade inicial da bateria.

\subsection{Modelo Cinético}

O Modelo Cinético de Bateria - do inglês, Kinetic Battery Model (KiBaM) - considera a carga da bateria distribuída em duas fontes: a fonte de carga disponível e a fonte de carga limitada, conforme apresentado na Figura 3. Observa-se que uma fração $c$ da capacidade total é distribuída na fonte de carga disponível e uma fração $1-c$ na fonte de carga limitada. A fonte de carga disponível fornece elétrons diretamente à corrente $i(t)$, enquanto a fonte 
Figura 3: Esquema de representação do Modelo KiBaM [15]

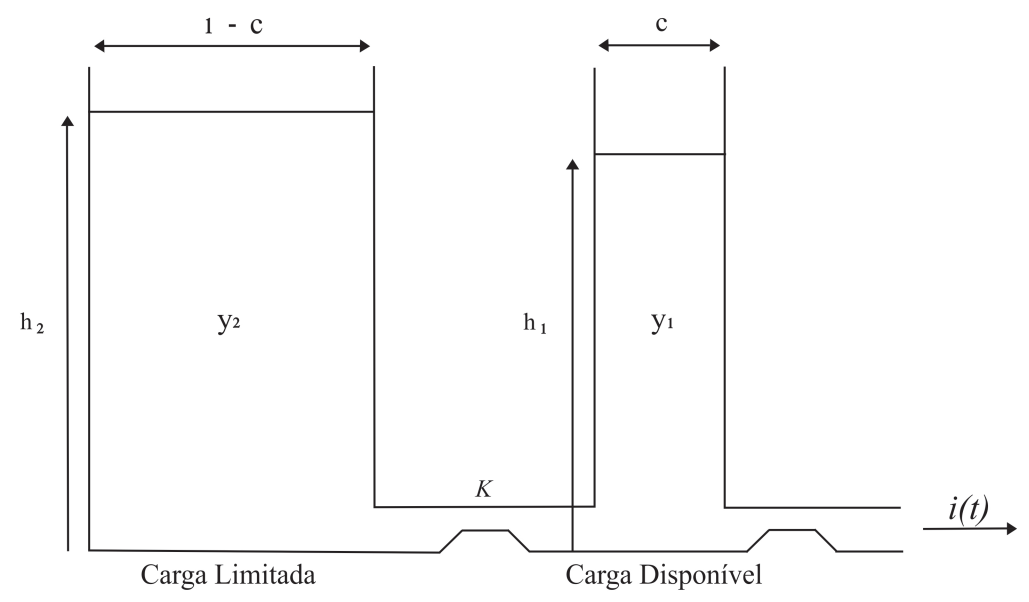

de carga limitada fornece elétrons à fonte de carga disponível - o parâmetro $k$ é a razão de fluxo entre as fontes [15].

Considerando $i(t)=I$ uma corrente constante, as equações que descrevem o modelo são:

$$
\begin{aligned}
& \frac{d y_{1}(t)}{d t}=-I-k\left(h_{1}(t)-h_{2}(t)\right) \\
& \frac{d y_{2}(t)}{d t}=k\left(h_{1}(t)-h_{2}(t)\right),
\end{aligned}
$$

sendo $y_{1}(t)$ a carga disponível e $y_{2}(t)$ a carga limitada. A altura em cada um dos tanques é dada por

$$
\begin{aligned}
h_{1}(t) & =\frac{y_{1}(t)}{c} \\
h_{2}(t) & =\frac{y_{2}(t)}{1-c} .
\end{aligned}
$$

Por simplificação matemática, uma nova razão constante $k^{\prime}$ é definida como

$$
k^{\prime}=\frac{k}{c(1-c)} .
$$

Substituindo as equações (8), (9) e (10) nas equações (6) e (7) tem-se

$$
\begin{aligned}
& \frac{d y_{1}(t)}{d t}=-I-k^{\prime}(1-c) y_{1}(t)+k^{\prime} c y_{2}(t) \\
& \frac{d y_{2}(t)}{d t}=k^{\prime}(1-c) y_{1}(t)-k^{\prime} c-y_{2}(t) .
\end{aligned}
$$

As equações (11) e (12) podem ser resolvidas aplicando-se as definições da Transformada de Laplace, com condições iniciais definidas por

$$
\begin{aligned}
& y_{1}(0)=c y_{0} \\
& y_{2}(0)=(1-c) y_{0},
\end{aligned}
$$


obtendo-se como resultado final

$$
\begin{aligned}
& y_{1}(t)=y_{1}(0) e^{-k^{\prime} t}+\frac{\left(y_{0} k^{\prime} c-I\right)\left(1-e^{-k^{\prime} t}\right)}{k^{\prime}}-\frac{I c\left(k^{\prime} t-1+e^{-k^{\prime} t}\right)}{k^{\prime}} \\
& y_{2}(t)=y_{2}(0) e^{-k^{\prime} t}+y_{0}(1-c)\left(1-e^{-k^{\prime} t}\right)-\frac{I(1-c)\left(k^{\prime} t-1+e^{-k^{\prime} t}\right)}{k^{\prime}}
\end{aligned}
$$

sendo $y_{1}(0)$ e $y_{2}(0)$ a quantidade de carga disponível e limitada, respectivamente, e $y_{0}=y_{1}(0)+y_{2}(0)$ a quantidade de carga total, no início do processo de descarga. Os parâmetros $k^{\prime}, c$ e $y_{0}$ são estimados a partir de dados experimentais [15].

\subsection{Modelo de Difusão}

O Modelo de Difusão de Rakhmatov e Vrudhula (i.e., Modelo RV), descreve o processo de difusão de espécies eletroativas, durante a descarga de uma bateria, com base nas Leis de Fick. Para isso, considera-se a evolução e a concentração dos materiais ativos na bateria durante o processo de descarga, modelando-a como um processo de difusão unidimensional em uma região finita [16]. Matematicamente, o modelo é expresso por meio de duas Equações Diferenciais Parciais (EDPs), dadas por

$$
\begin{aligned}
-J(x, t) & =D \frac{\partial C(x, t)}{\partial x} \\
\frac{\partial C(x, t)}{\partial t} & =D \frac{\partial^{2} C(x, t)}{\partial x^{2}}
\end{aligned}
$$

sendo que $J(x, t)$ é o fluxo das espécies eletroativas em função da distância $x \in[0, w]$ do eletrodo e do tempo $t \in[0, L], D$ é a constante de difusão, e $C(x, t)$ é a concentração de espécies.

Para uma bateria completamente carregada a concentração de espécies eletroativas é constante através do comprimento do eletrólito, disso decorre a condição inicial

$$
C(x, 0)=C^{*}
$$

sendo que $C^{*}$ é a concentração inicial de espécies eletroativas.

Conforme a Lei de Faraday, o fluxo em uma extremidade do eletrodo é proporcional à corrente de descarga, e na outra é nulo. Assim, para a descarga de uma corrente $i(t)$ e tempo $0<t<\infty$, tem-se como condições de fronteira

$$
\begin{aligned}
& \left.\frac{\partial C(x, t)}{\partial x}\right|_{x=0}=\frac{i(t)}{v F A D} \\
& \left.\frac{\partial C(x, t)}{\partial x}\right|_{x=w}=0
\end{aligned}
$$

sendo que $A$ é a área da superfície do eletrodo, $F$ é a constante de Faraday ${ }^{4}$, e $v$ é o número de elétrons envolvidos na reação química na superfície do eletrodo.

Aplicando-se o método da Transformada de Laplace e suas definições, utilizando a condição inicial e as condições de contorno, tem-se uma resolução para o sistema de EDPs (17) e (18), que relaciona o tempo de vida da bateria $t=L$, a corrente de descarga $i(t)=I$, e seus parâmetros empíricos, expressa por

$$
\alpha=\int_{0}^{L} \frac{i(\tau)}{\sqrt{L-\tau}} d \tau+\sum_{m=1}^{\infty} \int_{0}^{L} \frac{i(\tau)}{\sqrt{L-\tau}} e^{-\frac{\beta^{2} m^{2}}{L-\tau}} d \tau
$$

sendo que $\alpha=v F A \sqrt{\pi D} C^{*}\left(1-\frac{C(0, L)}{C^{*}}\right)$ e $\beta=\frac{w}{\sqrt{D}}$ são os parâmetros empíricos que dependem do tipo de bateria, eles são estimados a partir de dados experimentais [16].

\footnotetext{
${ }^{4}$ A constante de Faraday é uma constante física fundamental que representa a carga molar elementar. Atualmente, o National Institute of Standards and Technology (NIST) recomenda para a constante de Faraday o valor de 96485,33289 C mol ${ }^{-1}$ [17].
} 
Substituindo a equação (3) em (22), alterando os limites de integração, obtém-se

$$
\alpha=\sum_{k=1}^{n} 2 I_{k-1} A\left(L, t_{k}, t_{k-1, \beta}\right)
$$

sendo

$$
\begin{gathered}
A\left(L, t_{k}, t_{k-1}, \beta\right)=\sqrt{L-t_{k-1}}\left[1+2 \sum_{m=1}^{\infty}\left(e^{-\frac{\beta^{2} m^{2}}{L-t_{k-1}}}-\frac{\pi e^{-\frac{\beta^{2} m^{2}}{L-t_{k}-1}}}{\pi-1+\sqrt{1+\pi \frac{L-t_{k-1}}{\beta^{2} m^{2}}}}\right)\right] \\
-\sqrt{L-t_{k}}\left[1+2 \sum_{m=1}^{\infty}\left(e^{-\frac{\beta^{2} m^{2}}{L-t_{k}}}-\frac{\pi e^{-\frac{\beta^{2} m^{2}}{L-t_{k}}}}{\pi-1+\sqrt{1+\pi \frac{L-t_{k}}{\beta^{2} m^{2}}}}\right)\right] .
\end{gathered}
$$

A solução do conjunto de equações de (17) a (21) do Modelo RV, descrita pela equação (24), é obtida aplicando a Transformada de Laplace. Contudo, os parâmetros inicias do modelo ficam reunidos em apenas dois, $\alpha$ e $\beta$. A título de comparação de acurácia, é intuito desse trabalho determinar todos os parâmetros iniciais presentes nas equações do Modelo RV; para isso propõe-se uma solução geral alternativa que, por hipótese, pode ser escrita como

$$
C(x, t)=H(x, t)+V(x, t),
$$

sendo que $H(x, t)$ é uma solução particular do sistema, obtida por Tentativa Criteriosa, e $V(x, t)$ é a solução homogênea, obtida analiticamente pelo método de Fourier (i.e., Separação de Variáveis) [18].

Considerando, pelo método de Tentativa Criteriosa [18], que a função $H(x, t)$ seja descrita pela expressão dada por

$$
H(x, t)=a x^{2}+b x+c t,
$$

obtém-se, a partir da condição de fronteira (20), que

$$
\left.\frac{\partial H(x, t)}{\partial x}\right|_{x=0}=\left.\frac{\partial C(x, t)}{\partial x}\right|_{x=0} \Rightarrow 2 a(0)+b=\frac{i(t)}{v F A D} \Rightarrow b=\frac{i(t)}{v F A D}
$$

e da condição de fronteira (21), que

$$
\left.\frac{\partial H(x, t)}{\partial x}\right|_{x=w}=\left.\frac{\partial C(x, t)}{\partial x}\right|_{x=w} \Rightarrow 2 a(w)+b=0 \Rightarrow a=-\frac{i(t)}{2 w v F A D}
$$

e da equação (18), que

$$
\frac{\partial H(x, t)}{\partial t}=D \frac{\partial^{2} H(x, t)}{\partial x^{2}} \Rightarrow c=2 D a \Rightarrow c=-\frac{i(t)}{w v F A} .
$$

Substituindo a equação (26) em (18), (19), (20) e (21), com desdobramento algébrico elementar, obtém-se facilmente a EDP do problema homogêneo dada por

$$
\frac{\partial V(x, t)}{\partial t}=D \frac{\partial^{2} V(x, t)}{\partial x^{2}}
$$

e a condição inicial

$$
V(x, 0)=C^{*}-H(x, 0)
$$

e, ainda, as condições de fronteira homogêneas

$$
\left.\frac{\partial V(x, t)}{\partial x}\right|_{x=0}=\left.\frac{\partial V(x, t)}{\partial x}\right|_{x=w}=0
$$


A solução geral da EDP (30) é dada por

$$
V(x, t)=\frac{1}{2} K_{0}+\sum_{n=1}^{\infty} K_{n} e^{-\frac{n^{2} \pi^{2} D}{w^{2}} t} \cos \left(\frac{n \pi}{w} x\right)
$$

sendo que $K_{0}$ e $K_{n}$ são coeficientes de Fourier dados por

$$
K_{0}=2\left(C^{*}-\frac{a}{3} w^{2}-\frac{b}{2} w\right)
$$

$$
K_{n}=-\frac{4 a w^{2}}{n^{2} \pi^{2}}(-1)^{n}-\frac{2 b w}{n^{2} \pi^{2}}\left((-1)^{n}-1\right) .
$$

Das equações (26) e (33), substituídas na equação (25), para $i(t)=I$ e $x=0$, tem-se que a solução do conjunto de equações (17) a (21) do Modelo RV pela alternativa proposta é, portanto,

$$
C(0, t)=-\frac{I}{w v F A} t+C^{*}-\frac{I w}{3 v F A D}+\frac{2 I w}{v F A D \pi^{2}} \sum_{n=1}^{\infty} \frac{1}{n^{2}} e^{-\frac{n^{2} \pi^{2} D}{w^{2}} t},
$$

sendo $F$ a constante de Faraday, $I$ a corrente de descarga e $A, C^{*}, D, v$ e $w$ parâmetros do Modelo RV a serem determinados a partir de dados experimentais.

\section{Metodologia}

Nesta seção são apresentados os procedimentos metodológicos adotados para a aquisição dos dados experimentais, estimação dos parâmetros, simulação computacional e validação dos modelos. Os ensaios para coleta de dados são conduzidos no Laboratório de Sensores Inteligentes (LSI), do Grupo de Automação Industrial e Controle (GAIC), da Universidade Regional do Noroeste do Estado do Rio Grande do Sul (Unijuí).

\subsection{Coleta de dados}

A coleta dos dados experimentais, proveniente do descarregamento de baterias, é realizada através de uma plataforma de testes apresentada na Figura 4, desenvolvida por integrantes do GAIC. A interface de controle permite a rápida configuração dos experimentos e possibilita realizar até quatro descargas simultâneas, armazenando as informações de cada descarga em arquivos separados, facilitando a consulta dos dados. O gerenciador de descarga atua por meio de um microcontrolador, utilizando um algoritmo controlador Proporcional Integral Derivativo (PID), na gestão do perfil de descarga da bateria, adquirindo em tempo real informações de temperatura, tensão de saída e corrente de descarga.

Figura 4: Plataforma de testes para aquisição dos dados experimentais

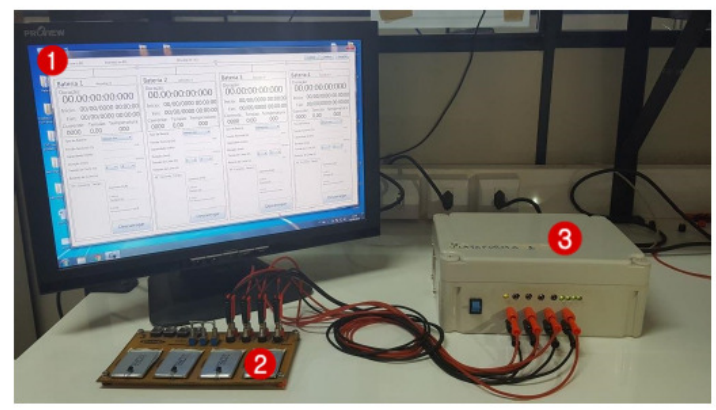

Legenda: 1 = Sistema de controle, 2 = Placa de baterias, $3=$ Gerenciador de descarga 
Para realização da coleta de dados é adotado um padrão único em todos os experimentos, com o intuito de minimizar alterações no resultado final dos testes. Inicialmente, as baterias são carregadas por completo utilizando um processo de carga no qual a bateria é conectada a uma fonte de tensão, recebendo uma carga lenta e constante de $20 \%$ da capacidade nominal, até atingir o valor de 4,2 V. Em seguida, com o sistema de controle configurado com os dados do perfil de descarga, inicia-se o processo que terminará quando a tensão da bateria atingir o nível de cut-off. Cabe destacar que os procedimentos são realizados seguindo as normas de segurança, além disso, a plataforma possui recursos de proteção que cancelam o procedimento de descarga ao sinal de falhas no sistema.

Os experimentos dividem-se em dois conjuntos de dados: correntes constantes e correntes variáveis. O primeiro conjunto, é utilizado para a estimação dos parâmetros dos modelos, sendo composto por 15 perfis de correntes de descarga constantes, que variam de $75 \mathrm{~mA}$ a $775 \mathrm{~mA}$, com intervalo entre os perfis de $50 \mathrm{~mA}$. O segundo conjunto é construído a partir da medição de correntes de descargas mensuradas em função das atividades realizadas em um smartphone, conforme Tabela 2, que combinadas geram 8 perfis de descarga variáveis. Destaca-se que o delineamento experimental segue em todos os ensaios as seguintes especificações, as unidades experimentais são baterias de Li-Po, modelo PL-383562-2C, sendo realizadas 8 repetições do ensaio para cada perfil. Observa-se que os dados coletados da plataforma de testes são apresentados na próxima seção para a melhor compreensão dos resultados obtidos.

Tabela 2: Principais correntes mensuradas em função das atividades de um smartphone

\begin{tabular}{lc}
\hline Tarefas & Corrente $(\mathbf{m A})$ \\
\hline Em stand by & 10 \\
Calculadora & 50 \\
Escrever mensagem & 70 \\
Câmera & 80 \\
Chamada normal & 100 \\
Visualizar imagem & 100 \\
Chamada em viva-voz & 150 \\
Acesso à $w e b$ & 200 \\
Rádio & 230 \\
\hline
\end{tabular}

\subsection{Estimação de parâmetros}

Neste trabalho utiliza-se o Método dos Mínimos Quadrados (MQ), que é um método de otimização que busca encontrar o valor ótimo dos parâmetros para um determinado conjunto de dados, através da minimização da soma dos quadrados dos resíduos, descrito pela seguinte equação

$$
V_{N}\left(\vec{\zeta}, Z^{N}\right)=\frac{1}{N} \sum_{t=1}^{N}(y(t)-\hat{y}(t \mid \zeta))^{2}
$$

sendo que $\vec{\zeta}$ é um vetor que contém os parâmetros a serem estimados, $Z^{N}$ é um vetor que contém as entradas e saídas experimentais, $y(t)$ são as saídas experimentais medidas, $\hat{y}(t \mid \zeta)$ são as saídas calculadas a partir do modelo que contém os parâmetros que se deseja estimar, e $N$ é o número de dados experimentais.

\subsection{Simulação e validação dos modelos}

As simulações são implementadas com auxílio do software de computação algébrica e numérica $M a t L a b^{5}$, em rotinas de execução $M$-File. Para estimar os parâmetros dos modelos é empregada a função de otimização lsqnonlin nas equações (5), (15), (16), (22) e (36), além dos dados experimentais das correntes de descarga constantes. A validação dos modelos se dá comparando os resultados das simulações com os valores experimentais dos perfis de corrente de descarga variáveis.

\footnotetext{
${ }^{5}$ Software comercial, utilizado sob licença Student.
} 


\section{Resultados e discussões}

Seguindo a metodologia apresentada na Seção 3, inicialmente são apresentados os dados extraídos da plataforma de testes e utilizados para a estimação dos parâmetros e validação dos modelos, considerando perfis de correntes de descargas constantes e variáveis. Em um segundo momento, são apresentadas as validações dos modelos matemáticos apresentados na Seção 2, confrontando os resultados encontrados a partir das simulações dos modelos com os dados experimentais obtidos da plataforma de testes. Por fim, é realizada uma análise comparativa entre os resultados dos modelos.

Os dados experimentais adquiridos via plataforma de testes, para o conjunto de perfis de correntes de descargas constantes, são apresentados na Tabela 3. A partir desses dados é realizada a estimação dos parâmetros empíricos dos modelos Linear, KiBaM e RV, através do método MQ. Os parâmetros encontrados foram os seguintes: para o Modelo Linear, a partir da equação (5), $C_{i}=46186,71$; para o Modelo KiBaM, a partir das equações (15) e (16), $k^{\prime}=37,46 \mathrm{~mA} \mathrm{m^{-1 }}{ }^{-1} c=1 \%$ e $y_{0}=47356 \mathrm{~mA}$ min; para o Modelo RV, via Transformada de Laplace, equação (24), $\alpha=2246,1 \mathrm{C}$ e $\beta=3,78 \mathrm{~s}^{-1}$; para o Modelo RV, via método de Fourier, equação (36), $A=2,5410^{-5} \mathrm{~cm}^{2}, C^{*}=4,2 \mathrm{~mA} \min , D=0,08 \mathrm{~mA} \mathrm{~s}^{-1}, v=4591,2 \mathrm{C}$ e $w=1 \mathrm{~cm}$.

Tabela 3: Dados dos perfis de descargas constantes utilizados para a estimação dos parâmetros dos modelos

\begin{tabular}{cccccccccc}
\hline I & L1 & L2 & $\mathbf{L 3}$ & $\mathbf{L 4}$ & $\mathbf{L 5}$ & $\mathbf{L 6}$ & $\mathbf{L 7}$ & $\mathbf{L 8}$ & $\mathbf{L m}$ \\
\hline 75 & 593,06 & 607,28 & 626,98 & 599,75 & 586,9 & 652,15 & 582,58 & 606,78 & 606,94 \\
125 & 388,48 & 392,93 & 375,03 & 367,4 & 380,9 & 393,65 & 381,55 & 398,17 & 384,76 \\
175 & 280,85 & 274,5 & 271,33 & 285,07 & 297,07 & 253,5 & 246,43 & 269,1 & 272,23 \\
225 & 206,45 & 191,38 & 188,35 & 207,45 & 199,83 & 211,8 & 214,07 & 208,58 & 203,49 \\
275 & 170,48 & 159,63 & 145,22 & 168,08 & 165,87 & 171,35 & 175,63 & 165,1 & 165,17 \\
325 & 141,57 & 145,68 & 149,53 & 143,47 & 148,45 & 129,85 & 126,4 & 145,33 & 141,29 \\
375 & 118,63 & 128,93 & 130,68 & 123,57 & 127,72 & 111,52 & 118,87 & 124,96 & 123,11 \\
425 & 111 & 113,72 & 94,47 & 110,13 & 105,3 & 109,03 & 114,4 & 109,01 & 108,38 \\
475 & 97,28 & 98,67 & 91,68 & 97,54 & 101,72 & 86,11 & 82,9 & 98,18 & 94,26 \\
525 & 88,57 & 91,3 & 93,15 & 85,28 & 92,63 & 78,67 & 74,11 & 85,85 & 86,2 \\
575 & 80,42 & 83,11 & 82,88 & 80 & 83,13 & 68,13 & 65,85 & 79,23 & 77,84 \\
625 & 73,6 & 74,38 & 77,58 & 72,4 & 77,3 & 62,42 & 59,83 & 73,12 & 71,33 \\
675 & 68,35 & 69 & 71,68 & 67,78 & 71,63 & 57,18 & 53,42 & 68,73 & 65,97 \\
725 & 63,53 & 63,77 & 66,68 & 62,27 & 66,42 & 51,62 & 48,27 & 62,98 & 60,69 \\
775 & 62,35 & 47,96 & 44,04 & 58,87 & 59,73 & 59,8 & 62,08 & 58,23 & 56,63 \\
\hline
\end{tabular}

Legenda: I = Corrente de descarga (mA); L1-L8 = Repetições; $\mathrm{Lm}=$ Tempo de vida médio (min)

Em seguida, definiu-se 8 perfis de descarga realísticos, apresentados na Tabela 4, a partir da medição de correntes de descargas mensuradas em função das atividades realizadas em um smartphone, conforme Tabela 2, da Seção 3. Ao contrário das descargas constantes, esses perfis possuem variações ao longo do tempo, então os efeitos não lineares estão mais presentes e, por consequência, o processo de descarga torna-se mais próximo ao perfil de utilização de um usuário. A partir da aplicação, na plataforma de testes, desses perfis de correntes de descarga variáveis foram encontrados os respectivos valores médios para o tempo de vida de cada perfil, que são apresentados na Tabela 5.

Tabela 4: Perfis de descarga variáveis

\begin{tabular}{ccc}
\hline Perfil & Corrente $(\mathrm{mA})$ & Tempo de execução (min) \\
\hline P1 & $100-10-150-10-100-10-200$ & $5-5-5-5-5-5-10$ \\
P2 & $250-400-50-200-550$ & $10-10-5-15-10$ \\
P3 & $750-450-200-150-250-100$ & $5-10-10-5-5-10$ \\
P4 & $100-200-300-400-500-600-700$ & $10-10-10-10-10-10-10$ \\
P5 & $700-600-500-400-300-200-100$ & $10-10-10-10-10-10-10$ \\
P6 & $170-270-10-140-230-10-270$ & $5-20-30-10-20-10-30$ \\
P7 & $270-10-120-170-10-270-170$ & $5-10-10-15-10-15-5$ \\
P8 & $200-10-300-10-200-10-400$ & $2,5-5-2,5-5-2,5-5-5$ \\
\hline
\end{tabular}

Revista Brasileira de Computação Aplicada (ISSN 2176-6649), Passo Fundo, v. 9, n. 4, p. 76-89, dez. 201786 
Tabela 5: Dados dos perfis de descargas variáveis utilizados para simulação e validação dos modelos

\begin{tabular}{cccccccccc}
\hline Perfil & L1 & L2 & L3 & L4 & L5 & L6 & L7 & L8 & Lm \\
\hline P1 & 463,81 & 512,01 & 470,10 & 490,25 & 463,33 & 494,11 & 470,91 & 473,20 & 479,68 \\
P2 & 146,53 & 147,68 & 156,13 & 149,15 & 146,80 & 148,48 & 147,98 & 152,28 & 149,38 \\
P3 & 143,68 & 144,90 & 143,96 & 145,69 & 139,96 & 139,96 & 136,53 & 139,35 & 141,76 \\
P4 & 135,49 & 133,50 & 125,56 & 124,68 & 124,23 & 122,68 & 120,00 & 126,79 & 126,62 \\
P5 & 99,21 & 105,73 & 99,93 & 97,00 & 95,98 & 99,21 & 93,31 & 97,71 & 98,51 \\
P6 & 265,38 & 263,61 & 321,45 & 264,56 & 263,86 & 319,26 & 270,50 & 310,88 & 284,94 \\
P7 & 340,23 & 330,50 & 308,86 & 313,81 & 304,16 & 333,60 & 331,00 & 303,93 & 322,01 \\
P8 & 327,48 & 352,51 & 326,28 & 327,55 & 326,03 & 319,95 & 311,78 & 301,75 & 324,17 \\
\hline
\end{tabular}

Legenda: $\mathrm{I}=$ Corrente $(\mathrm{mA}) ; \mathrm{L} 1-\mathrm{L} 8$ = Repetições; $\mathrm{Lm}=$ Tempo de vida médio $(\mathrm{min})$

Na Tabela 6, são apresentados os resultados das simulações, sendo que o Modelo Linear aqui é utilizado como base para a análise comparativa, pois é o modelo padrão utilizado pela maioria dos SGE dos smartphones atuais, o qual, neste caso, obteve um erro médio de 2,19\%. O Modelo KiBaM, por sua vez, obteve um erro médio de $1,84 \%$. Percebe-se ainda que o Modelo RV original obteve um erro médio de $1,86 \%$, enquanto o Modelo RV via Fourier atingiu um erro médio de 1,55\%; ambos predizem satisfatoriamente o tempo de vida de baterias de Li-Po, sendo que o Modelo RV, pela solução proposta neste artigo, alcançou um resultado ligeiramente melhor, em contraste com o erro médio da solução original.

Tabela 6: Resultados das simulações dos modelos

\begin{tabular}{ccccccccc}
\hline Modelo & \multicolumn{2}{c}{ Linear } & \multicolumn{2}{c}{ KiBaM } & \multicolumn{2}{c}{ RV } & \multicolumn{2}{c}{ RV-Fourier } \\
\cline { 1 - 4 } Perfil & Ls & Ea & Ls & Ea & Ls & Ea & Ls & Ea \\
\cline { 1 - 7 } P1 & 477,00 & 0,56 & 478,38 & 0,27 & 479,38 & 0,06 & 476,08 & 0,75 \\
P2 & 151,80 & 1,61 & 148,53 & 0,57 & 148,53 & 0,57 & 147,77 & 1,08 \\
P3 & 146,00 & 2,98 & 144,18 & 1,71 & 144,17 & 1,70 & 140,85 & 0,64 \\
P4 & 125,40 & 0,96 & 122,87 & 2,96 & 122,90 & 2,94 & 125,97 & 0,51 \\
P5 & 100,50 & 2,01 & 98,32 & 0,19 & 98,35 & 0,16 & 97,68 & 0,85 \\
P6 & 269,30 & 5,49 & 269,15 & 5,54 & 269,48 & 5,43 & 274,93 & 3,51 \\
P7 & 330,40 & 2,60 & 330,27 & 2,57 & 331,55 & 2,96 & 318,08 & 1,22 \\
P8 & 328,50 & 1,33 & 327,00 & 0,87 & 327,58 & 1,05 & 311,75 & 3,83 \\
\hline Erro médio & - & 2,19 & - & 1,84 & - & 1,86 & - & 1,55 \\
\hline
\end{tabular}

Legenda: Ls $=$ Tempo de vida simulado $(\min ) ; \mathrm{Ea}=$ Erro absoluto $(\%)$

Destaca-se, a partir do resultado das simulações, que quando o erro obtido do Modelo RV por Fourier é comparado com o erro obtido pelo Modelo RV resolvido através da metodologia tradicional, percebe-se uma diferença de $0,31 \%$, fato que implica em uma melhora de aproximadamente $20 \%$ em termos de acurácia. Além disso, o Modelo RV resolvido via Fourier possibilita a estimação de todos os parâmetros iniciais do modelo, ao contrário da metodologia original que realiza a simplificação dos parâmetros em apenas dois, $\alpha$ e $\beta$. Com isso, a extensão proposta fornece aos projetos que envolvem baterias, aporte ao desenvolvimento de novas tecnologias, a partir do estudo sistêmico de todos os parâmetros que compõem o Modelo RV. A otimização do tempo de vida de baterias, neste caso, pode se resumir à análise dos melhores parâmetros para compor um novo tipo de bateria.

Ainda a partir dos resultados da Tabela 6, observa-se que o Modelo KiBaM obteve em suas simulações computacionais um erro médio de $1,84 \%$. Assim, com o resultado obtido, o Modelo KiBaM é validado para predizer o tempo de vida de baterias de Li-Po, sendo uma alternativa matematicamente mais simples para predizer o tempo de vida de baterias [15]. Além disso, em perfis com menor possibilidade de ocorrer o efeito de recuperação (e.g., P4 e P6) o Modelo KiBaM apresenta erros maiores - o movimento é o contrário em perfis com maior capacidade de recuperação (e.g., P1 e P5). Isso aponta para o fato de que o modelo dá um peso maior para o efeito de recuperação, em detrimento da modelagem do efeito da taxa de capacidade. 


\section{Conclusão}

Neste artigo foram validados dois modelos analíticos - Modelo KiBaM e Modelo RV - utilizando perfis de corrente de descarga variáveis, para predição do tempo de vida de baterias. Seguindo a metodologia proposta para a pesquisa, com as simulações computacionais o Modelo RV original, obteve um erro médio de $1,86 \%$. Já o Modelo RV, via Método de Fourier, descreveu os dados experimentais satisfatoriamente alcançando um resultado ligeiramente melhor que a solução original, apresentando um erro médio de 1,55\%. Por fim, o Modelo KiBam alcançou um erro médio de 1,84\%, sendo validado para predizer o tempo de vida de baterias de Li-Po.

Os resultados apresentam um contraste ainda maior se comparados com o Modelo Linear de baterias, que obteve um erro médio de 2,19\%. Esse fato indica, apesar da evolução tecnológica dos dispositivos móveis, que os SGEs ainda carecem de melhorias no que diz respeito à acurácia preditiva do tempo de vida das baterias. A contribuição do presente trabalho se dá, primeiramente, em forma de incremento ao conhecimento técnico dos projetistas de smartphones, os quais têm a possibilidade de aperfeiçoar o SGE, a partir do emprego dos modelos aqui validados. Não só isso, mas também a contribuição se estende a própria validação dos modelos para aplicações em dispositivos móveis, uma vez que os modelos não foram desenvolvidos com foco em baterias de pequena capacidade.

Nesse sentido, acrescenta-se ainda que a partir dos resultados obtidos sugere-se como trabalhos futuros a implementação dos modelos em um sistema real (i.e., em um smartphone) para fins de gerenciamento estratégico de energia, além disso, pretende-se estender o estudo à otimização de parâmetros da bateria, para maximização do tempo de vida.

\section{Agradecimentos}

À Coordenação de Aperfeiçoamento de Pessoal de Nível Superior (Capes), pelo incentivo financeiro, à Universidade Regional do Estado do Rio Grande do Sul (Unijuí), pela estrutura disponibilizada, e ao Grupo de Automação Industrial e Controle (Gaic), pelo apoio técnico.

\section{Referências}

[1] TARKOMA, S. et al. (Ed.). Smartphone Energy Consumption: Modeling and Optimization. Cambridge, UK: Cambridge University Press, 2014.

[2] SIMPSON, R. Android Overtakes Windows for First Time. 2017. Disponível em: <http://gs.statcounter.com/ press/android-overtakes-windows-for-first-time>. Acesso em: 22 nov. 2017.

[3] SONG, C. et al. A novel electric bicycle battery monitoring system based on Android client. Journal of Engineering, Hindawi, p. 11, 2017. ISSN 2314-4912. Disponível em: <https://doi.org/10.1155/2017/2579084>. Acesso em: 22 nov. 2017.

[4] GARCHE, J. et al. (Ed.). Encyclopedia of Electrochemical Power Sources. Amsterdam, Netherlands: Elsevier B.V., 2013.

[5] GAZAFRUDI, S.; NIKDEL, M. Various battery models for various simulation studies and applications. Renewable and Sustainable Energy Reviews, Elsevier, v. 32, n. Supplement C, p. 477-485, 2014. ISSN 1364-0321. Disponível em: <https://doi.org/10.1016/j.rser.2014.01.048>. Acesso em: 22 nov. 2017.

[6] WESTERHOFF, U. et al. Analysis of lithium-ion battery models based on electrochemical impedance spectroscopy. Energy Technology, John Wiley \& Sons, v. 4, n. 12, p. 1620-1630, 2016. ISSN 2194-4296. Disponível em: <http://dx.doi.org/10.1002/ente.201600154>. Acesso em: 22 nov. 2017.

[7] FANG, H.; CAI, L. A summary of analytical model for lithium-ion batteries. International Journal of Science and Research, v. 4, n. 1, p. 2032-2035, 2015. ISSN 2319-7064. Disponível em: <https://www.ijsr.net/archive/ v4i1/SUB15702.pdf>. Acesso em: 22 nov. 2017.

Revista Brasileira de Computação Aplicada (ISSN 2176-6649), Passo Fundo, v. 9, n. 4, p. 76-89, dez. 2017 
[8] DENG, Z. et al. Online identification with reliability criterion and state of charge estimation based on a fuzzy adaptive extended Kalman filter for lithium-ion batteries. Energies, MDPI, v. 9, n. 6, p. 16, 2016. ISSN 1996-1073. Disponível em: <http://www.mdpi.com/1996-1073/9/6/472/htm>. Acesso em: 22 nov. 2017.

[9] TAO, L. et al. A review of stochastic battery models and health management. Renewable and Sustainable Energy Reviews, Elsevier, v. 80, n. Supplement C, p. 716-732, 2017. ISSN 1364-0321. Disponível em: <https://doi.org/10.1016/j.rser.2017.05.127>. Acesso em: 22 nov. 2017.

[10] MEYER, R. T.; DECARLO, R. A.; PEKAREK, S. Hybrid model predictive power management of a batterysupercapacitor electric vehicle. Asian Journal of Control, Wiley, v. 18, n. 1, p. 150-165, 2016. ISSN 19346093. Disponível em: <http://dx.doi.org/10.1002/asjc.1259>. Acesso em: 22 nov. 2017.

[11] ACPI. Advanced Configuration and Power Interface Specification 5.Oa. 2013. Disponível em: <http://www. acpi.info/DOWNLOADS/ACPI_5_Errata\%20A.pdf>. Acesso em: 22 nov. 2017. Acesso em: 22 nov. 2017.

[12] BUCHMANN, I. (Ed.). Batteries in a Portable World - A Handbook on Rechargeable Batteries for NonEngineers. Richmond, BC, Canada: Cadex Electronics Inc., 2016.

[13] VASCONCELOS, Y. Baterias Mais Eficientes. 2017. Disponível em: <http://revistapesquisa.fapesp.br/2017/ 08/15/baterias-mais-eficientes/>. Acesso em: 22 nov. 2017. Acesso em: 22 nov. 2017.

[14] PORCIUNCULA, C. et al. Avaliação comparativa entre o modelo elétrico Battery e os modelos analíticos Linear e Lei de Peukert. Revista Brasileira de Computação Aplicada, v. 4, n. 1, p. 71-80, 2012. ISSN 21766649. Disponível em: <http://seer.upf.br/index.php/rbca/article/view/1989>. Acesso em: 22 nov. 2017.

[15] JONGERDEN, M.; HAVERKORT, B. Battery Aging and the Kinetic Battery Model. Twente, Netherlands, 2017. Preprint submitted to technical report University of Twente. Disponível em: <http://wwwhome.cs. utwente.nl/ jongerdenmr/papers/battery_aging_kinetic_2017_02_23.pdf>. Acesso em: 22 nov. 2017.

[16] SILVA, B.; SAUSEN, P.; SAUSEN, A. Parameters estimation of the Rakhmatov and Vrudhula model from the optimization method search in improved network. ISRN Applied Mathematics, Hindawi, p. 9, 2014. ISSN 2356-7872. Disponível em: <http://dx.doi.org/10.1155/2014/958538>. Acesso em: 22 nov. 2017.

[17] NIST. Fundamental Physical Constants: Faraday Constant. 2015. Disponível em: <https://physics.nist.gov/ cgi-bin/cuu/Value?f>. Acesso em: 22 nov. 2017.

[18] BOYCE, W. E.; DIPRIMA, R. C.; MEADE, D. B. (Ed.). Elementary Differential Equations and Boundary Value Problems. San Francisco, CA, USA: Wiley, 2017. 